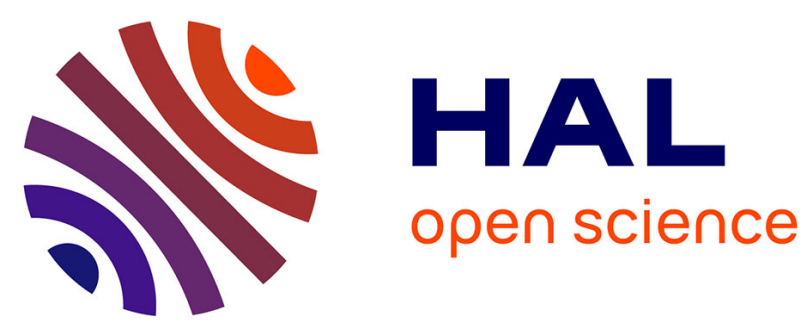

\title{
Multiscale modeling and imaging: the challenges of biocomplexity
}

Jacques Demongeot, Johanne Bezy-Wendling, Julian Mattes, Pascal Haigron, Nicolas Glade, Jean-Louis Coatrieux

\section{- To cite this version:}

Jacques Demongeot, Johanne Bezy-Wendling, Julian Mattes, Pascal Haigron, Nicolas Glade, et al.. Multiscale modeling and imaging: the challenges of biocomplexity. Proceedings of the IEEE, 2003, 91 (10), pp.1723 - 1737. 10.1109/JPROC.2003.817878 . inserm-00149851

\section{HAL Id: inserm-00149851 https://www.hal.inserm.fr/inserm-00149851}

Submitted on 9 Nov 2008

HAL is a multi-disciplinary open access archive for the deposit and dissemination of scientific research documents, whether they are published or not. The documents may come from teaching and research institutions in France or abroad, or from public or private research centers.
L'archive ouverte pluridisciplinaire HAL, est destinée au dépôt et à la diffusion de documents scientifiques de niveau recherche, publiés ou non, émanant des établissements d'enseignement et de recherche français ou étrangers, des laboratoires publics ou privés. 


\title{
Multiscale Modeling and Imaging: The Challenges of Biocomplexity
}

\author{
JACQUES DEMONGEOT, JOHANNE BÉZY-WENDLING, JULIAN MATTES, PASCAL HAIGRON, \\ NICOLAS GLADE, AND JEAN LOUIS COATRIEUX, FELLOW, IEEE
}

\author{
Invited Paper
}

\begin{abstract}
Computational modeling and imaging in biology and medicine are gaining more and more interest with the discovery of in-depth structural and functional knowledge at all space and time scales (molecule to proteins, cells to organs and organizms). The recursion between description levels for highly dynamical, interacting and complex systems, i.e the integrative approach, is a very challenging topic where formal models, observational tools and experimental investigations have to be closely designed, coupled and confronted together. Imaging techniques play a major role in this interdisciplinary attempt to elucidate this biocomplexity: they convey relevant information about the underlying mechanisms, depict the conformations and anatomical topologies and draw the biophysical laws they may follow. Furthermore, the basic image analysis tools (from calibration to segmentation, motion estimation and registration up to pattern recognition) are generic enough to be of value whatever the objects under consideration. The same comments apply when Computer Graphics or Virtual Reality techniques are concerned. This paper will survey the recent contributions dealing with both models, imaging data and processing frames. Examples ranging over different scales, from macro to nano, will be given in order to enhance the mutual benefits and perspectives that can be expected from this coupling.
\end{abstract}

Keywords-biological and medical imaging, interpretation, physiological modeling, simulation.

\section{INTRODUCTION}

The traditional image processing views rely most often on the application of innovative algorithms to clinical problems and, from there, it is expected that new clues will consequently follow in terms of health concerns [61]. It is argued in this paper that an in-depth knowledge of the processes underlying the observations will be more and more required to face the challenges of biocomplexity and will allow to visit and revisit a number of physiopathological issues thanks to the emergent imaging modalities at our disposal. There is a

The authors are with the University Joseph Fourier, Grenoble, University of Rennes 1, 35042 Rennes Cedex, France. condition, however, to succeed: to acquire a full pluridisciplinary culture in the area of concern. This statement will involve the introduction of our modeling capabilities, directly based on biology, physiology, chemistry and others, into the biomedical engineering space. Let us first define more precisely this issue.

Theoretical modeling and experimental investigations in the biomedical sciences are both aimed at the elucidation of the underlying biological or physiological processes, and one of the most critical features to deal with is the development and the evolution of complex spatio-temporal patterns, at different scales. Mathematical models for physiological pattern formation [54] have played a leading role in the formulation of hypotheses regarding possible mechanisms and in guiding the design of relevant experiments. From a general standpoint, modeling requires

- an in-depth understanding and a sound appreciation of the given problem: discipline frontiers have to be passed over;

- an adequate mathematical representation (continuous or discrete, qualitative or not, fine or coarse, etc.);

- the capability to generate a biologically-based or physiologically-based interpretation of the mathematical results in terms of expected behaviors, insights, and knowledge.

The need to merge knowledge coming from different sources about processes spanning several orders of spatio-temporal magnitudes is, at the same time, recognized as a critical step toward "integrative physiology." The aim is not only to store or retrieve the last research results and make them available to the worldwide community: it is also to provide operational tools for combining and confronting them to new modeling techniques which represent a unique way to make them active and to provide the right perspective for any understanding of large, multilooped systems. The recursion from cellular and subcellular levels to tissues 
and organs is here of major importance in order to build observable sets consistent with comprehensive views. Such objectives are stated by the "physiome" project $[5]^{1}$ and the "systeome" initiative ${ }^{2}$. This focus on in-silico models of whole cells, with fully-sequenced genomes, is similar to the effort initiated by the Alliance for Cellular Signaling(AfCS $)^{3}$ for cardiomyocytes and B-cells or to the Microbial Cell Project $(\mathrm{MCP})^{4}$.

These highly complex processes to be handled have also to be put in regards with the potential observables at our disposal for further validation. Imaging techniques are evolving in parallel and they bring new resources to explore the living structures and their functions. Beyond the classical ones, like CT Scanner and MRI, which are continuously improving, a wide spectrum of new physical means (fluorescence-optical, ion mass spectrometry, etc.) is available to acquire data on molecules and cells, in other words, to bring more insights at nano and micro levels. However, advances in imaging techniques will also strongly depend on the knowledge acquired about the biological mechanisms involved. Therefore, an increasing demand can be expected for active developments of algorithmic tools aimed at image analysis levels (segmentation, registration, motion tracking, deformation estimation, pattern matching, and recognition). We are convinced that a joint and proper use of sub and supra cellular knowledge, observational modalities and processing methods will offer a full range of unprecedented possibilities for diagnosis and therapy.

Some features of objects (i.e., structures and functions), imaging devices and models will be briefly examined Section II. Their coupling and confrontation as well as the transversal nature of information processing methods will be highlighted. The capability to draw well-posed questions from fundamental concerns or clinical objectives will be also addressed. Then, three examples will be described which operate at distinct object scales. The first one (Section III) deals with a generic approach capable to link textural features with tissue properties: it will be exemplified on a macroscopic model of the liver vascular trees coupled with CT Scanner data. Section IV is focussed on cell motion analysis observed by means of fluorescent confocal laser scanning microscope and, more precisely, on the phase corresponding to the nuclear envelope breakdown. The last example (Section V) is devoted to bio-array techniques allowing to provide some clues on the expression rate of genes. These examples underline the impact of a proper matching between models, data, and image analysis. Some perspectives will be given in conclusion.

\section{CONCEPTUAL AND INTEGRATED Views}

\section{A. Objects and Observations}

The targets range from molecules, proteins, genes, chromosomes, cells, substructures, organs, and systems up to

\footnotetext{
${ }^{1}$ http://www.physiome.org

${ }^{2}$ http://www.icsb2001.org/conference_schedule.html

${ }^{3}$ http://www.afcs.org/

${ }^{4}$ http://microbialcellproject.org
}

whole organisms and they have to be matched with imaging data (Fig. 1). The capabilities to extract, quantify, and interpret the information directly impact on the possibility to build, feed and validate relevant models. Diagnosis is the first objective and means that shape, tissue and function characterization will have a key role for the discrimination of diseases. However, diagnosis assessment is not the final goal and some therapeutic actions must be carried out such as surgery or radiotherapy strategies. They apply mainly to substructures and organs, both operating at a macroscopic level. They require a full framework of image processing methods. In addition to the image formation issue (including reconstruction algorithms, calibration, etc.), object extraction has to be performed which starts with the segmentation problem. In the medical context, the interindividual differences, the variability of disorder appearances, the inaccuracies and uncertainties in measurements are, among others, some of the intrinsic difficulties to deal with. This process requires the image volume or image sequence to be partitioned into regions representing meaningful objects. The current trend is to tackle this problem using some high level priors based on geometric and statistical models. Such models depart from the basic meaning used here: they can be considered as case-based constructs and do not refer to any underlying knowledge regarding tissue growth, disease evolution and the like. They can be identified as "surface models" [17]. Quantification is aimed at the characterization of the objects of interest like position, shape, tissue features, motion, deformation, etc., the outputs being numerical parameters that are assumed to fully define them as well as the relations they share with others. Registration techniques required for multimodal and interventional imaging can be derived from the same viewpoints. These issues are today well established and still represent an active area of research. We prefer to focus this section on more prospective topics tackling the cell level and below, and some of the corresponding imaging modalities.

They start from the acquisition of genomic or proteomic information to the modeling of both genes expression and metabolic networking which lead to complex spatio-temporal patterns and morphogenetic processes.

The main scientific interests, that can be expected in this area, are as follows:

- explaining morphogenetic mechanisms involved in embryology, tumor genesis and longevity processes;

- measuring gene expression, metabolic, and physical aspects of cells and tissues functions;

- investigating complex morphogenetic processes like tumour progression, neo-vascularization, wound healing;

- modeling cell and tissue dynamical organization (proliferation, apoptosis, migration, remodeling, etc.) based on the evolution of highly orchestrated interactions and complex intra/extra cellular signalling pathways;

acquiring, processing, and interpreting images from various technologies such as cDNA-, protein-, 


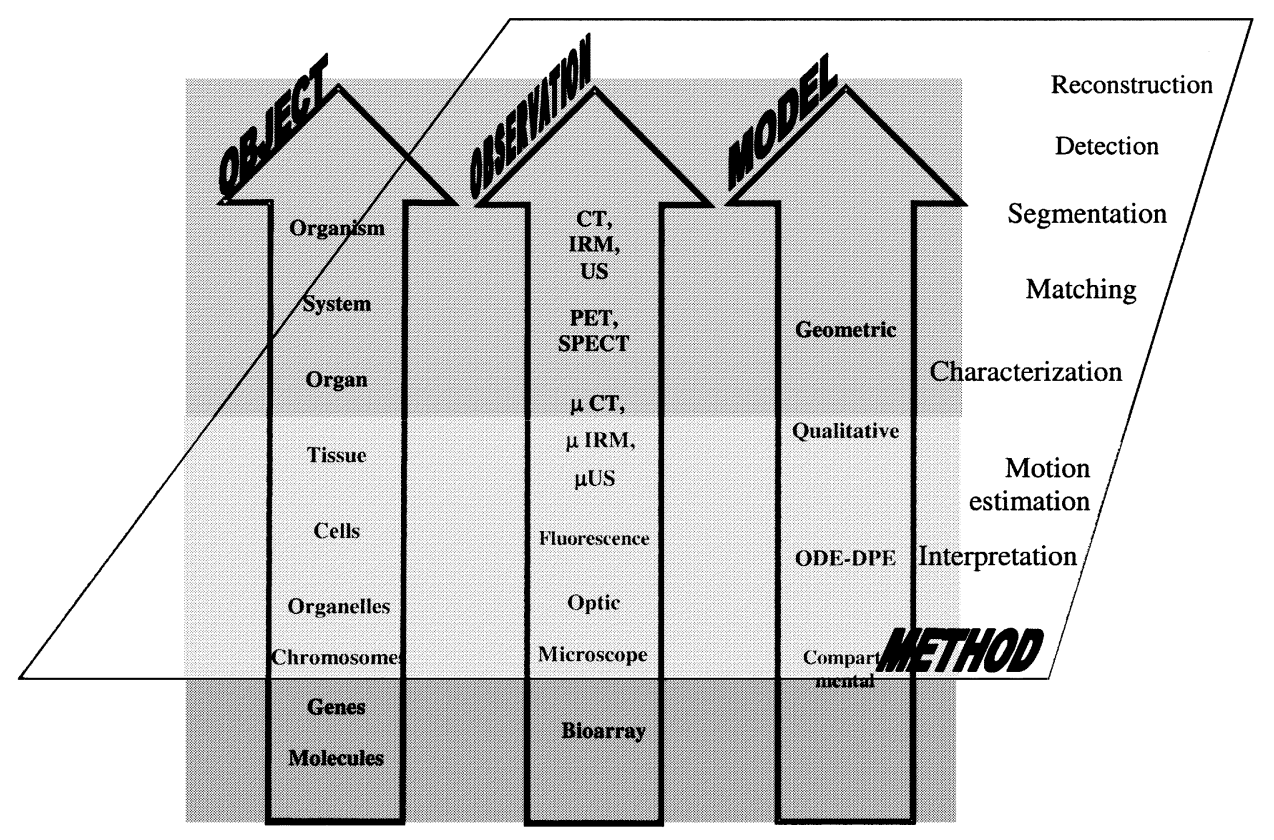

Fig. 1. Objects-observations-models. These basic dimensions, explored at different scale levels by means of appropriate imaging modalities and models, can be submitted and coupled to generic components of information processing in order to gain in-depth understanding about normal and pathological features.

tissue-arrays, image cytometry, or two-dimensional gel electrophoresis;

constituting databases and patient centred data warehouses allowing diagnosis and predictions in familial or population contexts.

\section{B. Multilevel Models and Imaging}

Any advance in understanding the cell functioning must be formalized by finding a high level of formal description of biochemical, mechanical, signalling processes used in cell biology, from the genome expression to cell motion and tissue morphogenesis. This close coupling between data, knowledge and models will allow us to come up with missing elements ensuring the coherence and the pertinence of the explanation of observed pathological phenomena in order to increase the efficiency of further medical actions. A bridge between the cell and molecular level, on the one hand, and higher levels of organization, on the other hand, has to be built. The elucidation of these interlevel processes relies on structural and functional information which includes membrane features, channel kinetics, transporter characteristics, metabolic network topology as well as tissue properties (architecture, elasticity, fluid dynamic, etc.).

Such an ambitious perspective emphasizes that the future, and major challenge, in medicine is to move from diagnosis to disease prediction and prevention. It requires new means to detect very early the onset of a given pathology. The recent International Symposium on Biomedical Imaging (ISBI) [74], has shown that fluorescence imaging in optical diffusion tomography, near-infrared optical tomographic imaging, real-time and functional optical coherence imaging, soft X-ray microscopy among others [6], [25], [30], [39], will play a major role. DNA chips, Biochips, and Molecular Imaging will provide the key elements to reach such understanding. Molecular Imaging [33], [56], [76] can be seen as the capability to get a localized in vivo characterization of biological processes on the molecular and cellular level. In contrast to the morphological and functional changes that are caused by a disorder through conventional imaging, specific molecular abnormalities could be detected which are the cause of the disease. It relies on a transfer of methods developed in molecular biology and in in vitro diagnosis to imaging where the key elements will be the design of specific and biocompatible probes with high specificity, appropriate radiotracers and fluorescent dyes, amplification strategies (e.g using reporter genes in gene therapy for instance [56]) and sensitive detection systems.

While CT Scanner and Ultrasound techniques provide morphological and physiological information, magnetic resonance imaging (MRI) and spectroscopy (MRS), nuclear imaging (PET, SPECT) and optical modalities bring clues on metabolism and molecule distribution. They differ, however, in their capability to capture these processes. The spatial resolution of PET Scanner can be expected to decrease beyond $1 \mathrm{~mm}$ [37] but requires an increased sensitivity, higher specific activity labeled compounds and improved reconstruction algorithms [26].

MRI [45] provides access to host intrinsic parameters, including molecular motions (such as diffusion and perfusion), susceptibility effects and chemical shift differences. Enzymatically activated contrast agents have been already reported [47] which allow in vivo visualization of exogenous gene expression and this points out the MRI potential for cell therapy and gene therapy.

Fluorescence-optical imaging with biologically activated contrast agents is limited by the penetration depth (about 
$7-12 \mathrm{~cm}$ according to the type of tissues [55]). However, this modality is highly sensitive to molecular information, fast and cost effective and does not involve ionising radiation. The objects close to the surface can be imaged directly and depth information can be obtained by means of tomographic techniques (it has been shown recently that deep brain tumours can be detected [76]). Near-infrared fluorescence imaging is also of interest when contrast agents can be switched on by enzymes of tumour metabolism (so-called "smart contrast agents"): an improved signal-to-noise ratio is provided. It can be imagined, even if it is far away, that virtual biopsy could be achieved instead of invasive tissue sampling and histology: the molecular characterization of disease in situ for personalized treatments of patients and the earlier determination of therapy response would then be possible.

Multiple isotope imaging mass spectrometry (MIMS) allows to study the accumulation of molecules labeled with stable isotopes $(15 \mathrm{~N}, 2 \mathrm{H}, 33 \mathrm{~S}$, etc.) or radio-isotopes $(14 \mathrm{C}$, etc.) in a given target [63]. The image resolution $(50 \mathrm{~nm})$ is better than optical microscopy (about $0.3 \mu \mathrm{m}$ ). It has a wide spectrum of biological applications from histological image to halogenous tracer mapping: cellular metabolic pathways, nucleo-cytoplasmic translocations, RNA and DNA expression, and distribution can be observed.

Of course, as it can be already seen in medical equipment (CT-PET scanner), some of these sources may be combined in a unique device, with the major advantage to bring complementary information and to avoid the image registration task.

\section{Models and Information Processing}

Modeling belongs in a general sense to cognition and can be seen basically as a predictive science. Model-driven plans can be found for data acquisition and are aimed at the optimization of measures. Model-driven experiment design in order to test hypotheses is another example of this. Conceptual modeling and planning are closely related together: the former helps to describe the world in which to operate, the latter provides a framework to organize the actions and anticipate on potential evolutions. This is of significance when dealing with computer assisted therapy ([67]). However, modeling is not limited to a descriptive role but has a major interest in providing solutions to the problem at hands. It is expected to improve the resolution of a problem by introducing explicitly current knowledge in the reasoning and decision processes. This applies to low level tasks (capture of information, control of processing algorithms), intermediate level actions (feature grouping, focus of attention techniques, hypothesis generation,...) and to high level analysis (diagnosis, corrective therapies. etc.). When intelligent systems are concerned, explanations and causal relations are expected to be provided together with the solutions.

Numerical simulations bring us closer to the core of this paper. They have to see with key research issues such as the paradigms regarding direct and inverse problems, image analysis and synthesis, etc. Simulation relies on a model of the physiopathological mechanisms to be studied and its first goal is to realistically reproduce the observed behaviors. It should be stressed that such mathematical descriptions of processes are not aimed at explanations. They are used to have more insights or understanding of a system, as a prior step before experimentation on real data but also when it is impossible for ethical reasons or technical constraints to make these experiments. Simulations have a direct link to the recent trend of virtual reality in medicine where training and planning in preoperative situations, and intraoperative guiding during computer-assisted interventions are central elements.

Simulation can be obviously complemented by the more ambitious goal of identification which deals with both the system structure and the unmeasurable variables (estimating the internal parameters and variables of physiological interest). From that, prediction and control can be derived or tracking the administration of therapeutic agents can be achieved. Theoretical open problems however are still remaining due to the fact that the system complexity may be high and the observables are often sparse. System identification belongs to the general class of inverse problems previously mentioned: it still received a limited attention from the signal processing community despite the proximity of concepts and tools with automatic control. Inverse problems in biomedical engineering have been mainly addressed in image reconstruction [34] and brain or cardiac source identification in EEG or MEG recordings.

\section{Models and Biocomplexity}

Any mathematical study of physiopathological processes is first challenged by the intrinsic complexity to deal with and by the fact that numerous of mechanisms are still ill-understood (i.e the active cellular controls or the large loop regulations such as the autonomic nervous system for instance). What can models do then? It is argued that they will be useful even in case of a complete understanding of the processes by the capabilities they offer to explore the influence of changing the variables associated to particular scenarios, to compare the outputs to the observations and to detect sensitive parameters (in particular those causing dramatic changes in the number or nature of stationary, periodic, or chaotic states). The hope behind is also, by establishing model parameter deviations from normal behaviors (structural changes may clearly occur which are much more complicated, in comparison with parameter control, to deal with), to conjecture the reverse process, in other words to derive ways to go back from abnormal to normal states. There is certainly a long way to follow before being able to reliably reproduce the multitude of physiopathological behaviors despite the theories that abound. Basic mechanisms and interactions involve a large number of variables acting at different levels of organization (molecule, gene, protein, cell, organ or individual, population, etc.) as well as strong reciprocal influences with the environmental conditions. The understanding of such complexity, from very local entities to large organizations, with all the required updates which can be assumed, can be only achieved by theoretical modeling. This has motivated the emergence of the "integrative 
physiology" concept which, if limited to the accumulation of knowledge advances coming from different fields of concern, will have a poor impact. Electrical mechanisms (excitation, propagation), biomechanics (contraction/expansion/deformation estimation from motion in dynamic CT, elastography form ultrasound), haemodynamics (coronary blood flow imaging), etc. whatever the description level they convey, will never be sufficient as such to get an integrated view of the heart. When put in relations together, then, a real breakthrough can be expected and models here can play a major role for diagnosis and therapeutic assessment. By first describing the dynamics of the relevant variables at each level and then by modeling the interactions between them, a more complete understanding of the corresponding processes can emerge at higher levels or be questioned at lower levels. This can be seen as the essence of integrative physiology: a path from microscopic processes to global properties at macroscopic levels of organization.

The models that have been suggested so far can not afford any completeness. They provide some insights on different aspects of the physiological processes. The study of the robustness of generated patterns to small (random or not) modifications of the initial and boundary conditions, to structural changes and the joint parameter variations, is of critical importance.

Ordinary differential equations (ODEs) are well suited to render dynamical behaviors of individual entities or small size organizations, but they become intractable for large assemblies and, because of their intrinsic non linearity, relevant physiological equations for populations can not be derived in a direct way from the equations governing elementary components. In other words, models of individuals are not matching models of assemblies. Alternative options to ODE systems should also be examined.

To summarize this section, it can be said that modeling as such is not enough: it must be confronted and matched to experiments and observables. It is not advocated here that a physiological model must be either the first step to accomplish in biomedical image processing or the ideal solution to the problems at hands. Explicitation of simple properties and assumptions about structures and dynamics twinned with proper data models, in other words, surface models (i.e., not referring to physiological mechanisms, for instance textural features) may provide better prediction and decision capabilities. Such external approaches, however, remain descriptive and physicians may have some reluctance in using them because no link is established with their knowledge.

\section{E. Modeling Issues}

Most of these issues apply to all classes of models whatever their purposes. The ways they are answered, when possible, are however depending on the nature of the models. They correspond mainly to important question marks and consequently to challenging problems to be solved, not to methodological answers [13]. Some are of concern only for mathematical models and of interest in the special case of closed-loop systems (i.e., coupled with observables): a key property is identifiability.

The plausibility of a model can be appreciated with regard to the realistic features (architecture and functioning) included into it. Its evaluation can be performed only with respect to another one: for instance, by the spectrum of situations that could be generated or by the amount of knowledge it incorporates (but knowledge, its use and confidence, make any quantification difficult). It could be related also to the notion of completeness. A theoretical demonstration or proof that a model would be distinguishable from another is also desirable. However, other issues are equally critical. Is it possible to make a model minimal or parsimonious? The robustness of a priori knowledge (including parameter setting and impact) and of constraints (introduced for instance through regularization techniques) have also to be estimated. The capability to integrate and merge exogenous data or information (clinical symptoms, electrical data and imaging examinations), is of high relevance but, with the exception of knowledge-based systems or statistical approaches, none of the theoretical models at our disposal is able to handle such requirements. The same comment can be done when heterogeneous model fusion or coupling (i.e qualitative or symbolic with numerical models) is considered. In addition, the combinatorial space to be faced, the nonconvex functionals to be minimized in some models lead to mathematical problems independently of the physiopathological concerns.

Multiple factors will affect the recorded data (sensor influence, noise, nonstationarity, etc.) and will directly impact on model identifiability. In addition, in some cases, strong restrictions appear on the type and the modalities of measurements. This is the case of compartmental models, applied to many functional imaging sources (MRI, SPECT, and PET), which have been specifically and extensively studied [38]. Important notions have been established regarding $a$ priori, local and global identifiability for linear and nonlinear systems. Identification techniques for compartmental models may apply only on specific cases: for instance least square, maximum likelihood when the model is uniquely identifiable or, otherwise, derivations of bounds, parameter aggregation, model reduction can help in estimating some of the parameters. Recent contributions have focussed on differential algebra for nonlinear model identification [3], [46] but they are limited to low order models. Not only complex models are inherently representative of physiopathological processes, but free noise data and error-free model structures are difficult to assume. The example reported Section III will however show that, a fairly complex model can be useful to better apprehend the underlying role of vascular components in the image contents. Any model, in between external descriptions and physiologically based models, has a role to play from research to clinical applications as far as it is associated to a right objective and a sound validation.

Of course, other issues are coming up when a modeling operation is launched. The selection of a model class (hidden Markov hodels, ODE, qualitative models, etc.) is among the early steps. There is no objective rule to do that and no class is capable to claim genericity and superiority. 
Competition between approaches should be the basic principle but, unfortunately, the absence of communication and crossfertilization between disciplines most often prevents any comparison. It can be thought that the nature of both the observables and the knowledge at our disposal, the capability of a class to represent the processes and the formal properties which are attached to it (from identifiability to inversion problems), the time and space dimensions in which to operate, strongly condition this choice. Another critical issue is the description level (or scale) which highly influences the complexity of a model. This problem is identified under very different paradigms (granularity, surface/deep, fine-to-coarse, local/global, detail level) according to the field in which they have been used. It concerns time and space scale selection as well. A simple example can be found for spatial qualitative relations in three-dimensional worlds ("object in front of," "moving backward," etc.) as opposed to numerical features (position, orientation described by means of a geometric coordinate frame). A possible rule to specify this scale could rely on the tuning between three entities: i) the level of details or decomposition of the model; ii) the scale of observations; and iii) the algorithmic processing capabilities at our disposal (i.e, the quality of the information that it is possible to extract). Here also, the modeling purpose is of great importance. Simulation will give more degrees of freedom (large scale, distributed systems) when compared to identification where higher aggregation is required.

The validation of models is also a complicated task in modeling physiological systems. It means to estimate whether or not a model fits with its purpose. It has been already said that properties like plausibility and quality of a model are difficult to formulate. Establishing the validity range, either for simple or complex models, can be performed on cases, learning sets, experimental data matching, expert confrontations, exception studies, etc., but an objective, widely accepted, measure is difficult to set. Its assessment can rely on its theoretical consistency, its robustness to changes or noises, the deviations from experimental data, its testability (protocols of practical tests) but can be appreciated through its utility (assistance to a decision, capacity of prediction or conjecture formulation). It must be understood for instance that complex models are inherently incomplete, with a high degree of imprecision and uncertainty on the structure and the parameters, and consequently more difficult to validate.

In other words, no ground truth can be established for living systems and diseases. Performances of biomedical image processing algorithms are equally difficult to assess for the same reason. A progressive scheme may rely on phantom design, fully simulated data (without any link to the underlying mechanisms), hybrid data (a mixture of synthetic data and real data) and real images with expert annotations. The ground truth is therefore partially controlled but its realism can be still questioned. Symmetrically, model-based simulations can be performed, statistical features measured on the generated images and compared to their equivalent on real data. This idea shows how the image processing can be closely linked to modeling for validation purposes.

\section{F. Biomedical Triangle}

The all stages depicted Fig. 1 are evolving very fast and a full review will go beyond the scope of this paper. Two examples of methods related to extraction and quantification in images are mentionned below which brought seminal contributions. Deformable and functional models, inspired by the physics of flexible materials [68], [69], evolve according to forces derived from the image data and can be used to fit, reconstruct, match and track objects when some salient features like edges are present along the boundaries of these objects. In the level set approach [62], a curve (or a surface) representing the boundary of a structure is embedded as a zero level of a higher dimensional surface. Some energy-based active contour minimization problem is solved by computing the geodesics or minimal distance curves. Because local and global information is used, robustness to weak edges can be achieved. Numerical stability and topological flexibility are also one of the most interesting properties of this approach. Structured deformable models composed of a group of basic shape elements have also been proposed. They follow a growth process controlled by the morphogenesis [36]. All these methods belong to the wide field of computer vision and they are of value in medical imaging as well as in traffic analysis, indoor navigation, remote sensing detection, etc. They are generic enough to deal with the several objects, images and tasks which have been sketched above provided that some adaptations are made. In other words, image analysis methods, but also computer graphics techniques for visualization purpose, apply to the multiscale objects and observations of concern. This justifies their transversal positioning and displays the active role they have when a cooperation with models are looked for. However, as pointed out in Fig. 2, imaging modalities and algorithmic resources are not enough: the combinatorial explosion that exists behind the objects can only be faced through well posed questions. This is not an easy task because it assumes a sound knowledge of the all facets of a given biomedical problem. The triangle and the bidirectional arrows in Fig. 2 exemplifies the partial space into which relevant and operational views have to be built. For instance, in-silico simulations of cell or organ behaviors can complement in vitro and in vivo studies: going from a medical problem of pathologic morphogenesis to the knowledge mining and modeling, passing through the data acquisition. The need for a language of description of the many different entities like cell functions, intra- or intercellular interactions, localization (growth and motion), signalling, referring to cell physiology, systems biology and cell anatomy is a major concern. Such language will allow the implementation of relevant algorithms that are able to simulate functions, interactions, localization and signalling by using hybrid models (e.g., Boolean systems for the genomic interactions, fixing the values of parameters of partial differential systems whose attractors represent the final morphologies to be explained).

The three examples illustrated in the following sections do not pretend to cover this wide spectrum: they just highlight 


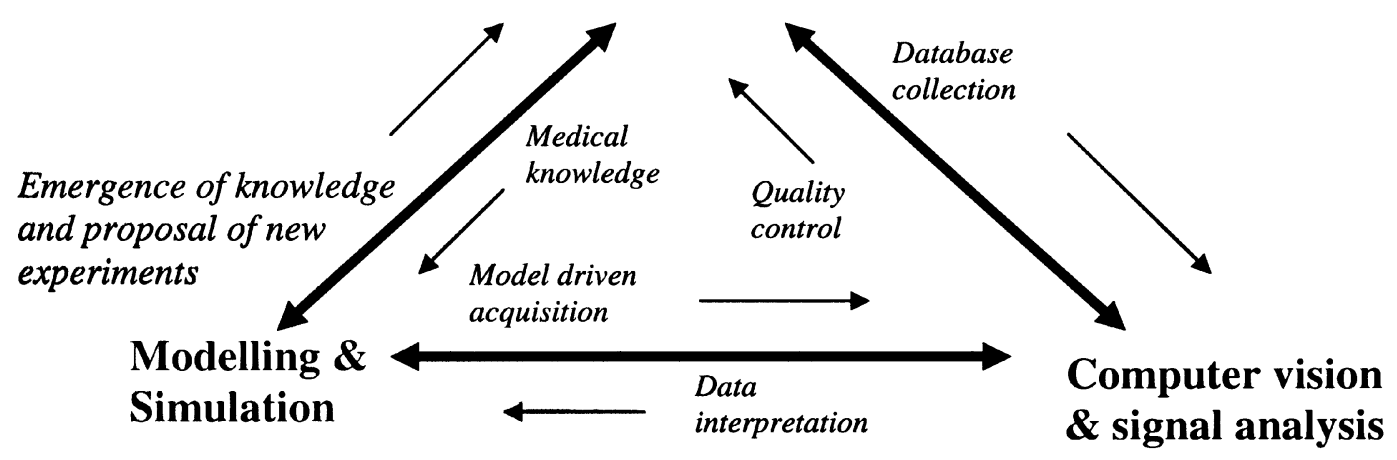

Fig. 2. Interrelations depicted by this triangle point out the diverse facets to deal with patient diagnosis and therapy, from medical knowledge, quality control up to database collection.

situations encountered along the object scales, the role of modeling and the transversal place of image analysis methods.

\section{MACROSCOPIC MODEL: APPLICATION TO THE LIVER VASCULAR NETWORK}

A generic scheme is described Fig. 3. It can deal with the all features of relevance for an in-depth model-based representation, the "object space," from structural and functional properties to the deviations that can occur during the organ formation or after (reflecting inter-individual variations as well as pathological evolutions). The sensing device, specified here as "virtual imaging system" must also be modeled in a realistic way in order to simulate valuable images. This task involves to take into account the physical principles of the imaging modality and the image reconstruction algorithms (both being merged into the "sensor space") leading to image formation. Any imaging device can be considered according to the organ properties to be analyzed. Modeling "object" and "sensor" is very challenging as such but it is the only way to get a really relevant "image space." The virtual data so produced can then be submitted to any image analysis method. Such processing can be oriented toward morphological or structural studies and focussed, among many others, on segmentation and characterization tasks [static two-dimensional (2-D) or three-dimensional (3-D) images]. They can deal with functional features when time stamped images are generated (i.e., time image sequences) using motion estimation or compartmental models when they are of concern. The overall system output belongs to what is called the "decision space", which may rely on any pattern recognition methods (statistical data analysis, neural networks, etc). From this standpoint, an equivalence can be established between the "virtual space" and its dual, the "physical space."

An instantiation of this conceptual approach is presented in this paragraph, where the object is then the vascular network of a given organ, the liver, which is modeled at each step of its development, with structural, morphological and functional information. The virtual sensing device is a standard CT Scanner providing 3-D observations and, in simulation, time image sequences when blood flow is considered. The image analysis deals with both detection problems (testing how far the most advanced methods can go to extract small vessels for instance) and texture characterization (emphasizing the need for feature meaning). These issues will be detailed in Sections IV-VI.

The 3-D model concerns two main components: the tissue and the vascular network that perfuses it and adapts to its local geometry. 3-D models have already been proposed to simulate the heart vascularization [40]. In the model we propose, the process starts with an organ (here the liver), whose size is a small fraction of the one of an adult organ, and whose growth continues until it reaches its full size. The changes in the size and structure of the organ and the corresponding vascular trees operate at discrete time instants (cycles). The organ is constituted of "macroscopic functional units" (MFUs) evenly distributed. Each MFU belongs to a class, characterized by functional and structural properties (e.g., minimal distance between MFUs, probability of mitosis/necrosis) and physiological features (e.g., blood flow rate or corresponding pressures). Several classes of MFUs can co-exist in the model, leading to regions with different functional properties. For instance, an hypervascular tumor can be simulated by introducing, among the normal MFUs, some abnormal ones, which lead to local vascular modifications. The tumor has only an arterial blood supply and does not receive portal venous blood flow, when normal hepatic tissue is perfused by the three hepatic trees (Fig. 4). Moreover, probability of mitosis, maximum local density and blood flow are increased in the pathological case.

In Fig. 5, CT scans simulated with variable acquisition parameters (spatial resolution, slice thickness, arterial or portal phase) are presented. The method is based on projections/filtered backprojections computation and take into account the partial volume effect [43]. These images are located in the tumoral part of the liver. The lesion appears hyperdense compared to the normal surrounding parenchyma during the arterial phase, and slightly hypodense during the portal phase [8], [9]. This temporal evolution is in agreement with 
Object Space Sensor Space Image Space Decision Space

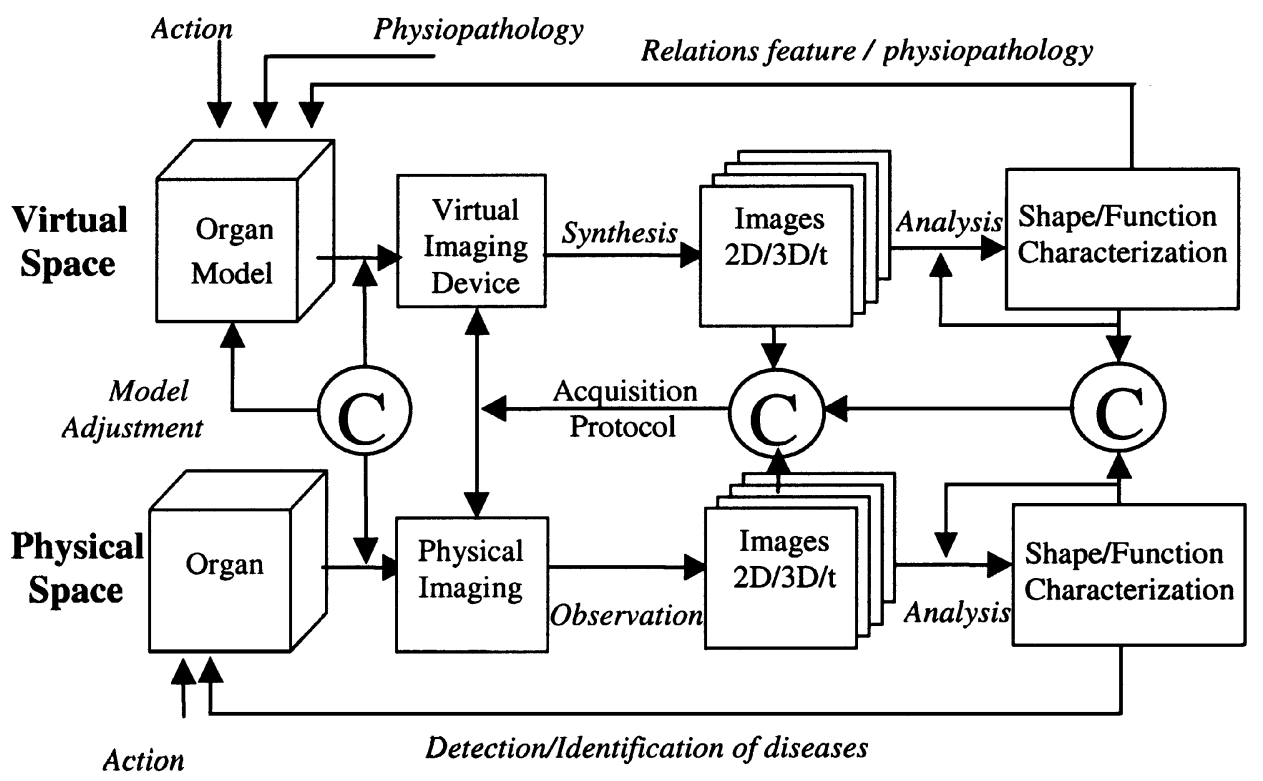

Fig. 3. Generic model-based approach for medical image analysis. The decomposition into virtual/physical spaces and object/sensor/image/decision spaces allows us to precisely identify the several intervening components in place and to define the relevant matching stages for comparison and validation (depicted here by $\mathrm{C}$ ).
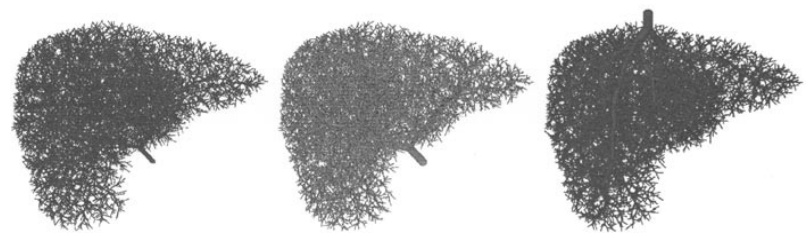

Fig. 4. Simulated hepatic vascular network with an hypervascular tumour. (Left) Hepatic artery (HA). (Middle) Portal vein (PV). (Right) Hepatic vein. The tumor is supplied only by the artery and not by the portal vein (it corresponds to a region with a higher density of vessels in the left and right pictures).

the complex hepatic enhancement observed in in vivo examinations [4], [28]. During the first early acquisition phase (around 20-30 s after contrast infusion), the hypervascular lesion receives highly concentrated dye product coming from the HA, while the liver parenchyma is less enhanced because receiving less CM (PV does not contain CM yet), which leads to a high lesion conspicuity. In a second phase, the normal parenchyma enhances a lot, due to the important flow of CM arriving through the PV (representing around $80 \%$ of the total flow). The parenchyma enhancement becomes comparable (hypo or iso-density) to the tumor's one, leading to a decreasing conspicuity. This global behavior can be further analyzed by compartmental methods, both in the virtual and the physical spaces, and the results compared for validation purpose.

Fig. 6 displays the evolution of the textural feature Gray level distribution [12], [13], derived from the run-length matrix with time, slice thickness, and zoom, in the normal and the pathological situations. The two surfaces, describing the evolution of this feature, intersect in a similar way at both phases. These results confirm that different acquisition con- ditions of CT scanning influence strongly the texture observed on images. They also point out that textural features can potentially be used for discrimination purposes in clinical examinations, but only when the same (or at least similar) protocols are used. This model, as well as other models of enhancement [48], can also be useful to study the injection and the acquisition times (arterial phase, portal phase), in order to optimize the lesion detection and characterization [24].

Using this example and coming back to Fig. 3, a number of highly meaningful concepts can then be projected onto this $2 \times 4$ matrix. A few are exemplified here. The first one corresponds to the capability at each step to compare the real and virtual outcomes either at a visual level or through quantitative characteristics. For example, local and global statistical measures (the above textures, but also distances, volumes, etc.) can be performed on the simulated as well as on in vitro or in vivo data. They can bring new clues for model building and adjustment (initialization conditions, error criteria, etc.).

The second one emphasizes the possibility to directly link the decision space (and the extracted features) to relevant physiopathological patterns: this opens the road toward physiologically founded understanding of image features. Direct or inverse problems are here of main interest if it can be proven that the corresponding system is identifiable. However, a pragmatic solution consists to estimate the linear or nonlinear relations between the measures and the underlying, fully controlled, physiological variables as it has been shown Fig. 6.

Moreover, through these simulation capabilities, the impact of changing the acquisition parameters (for instance 


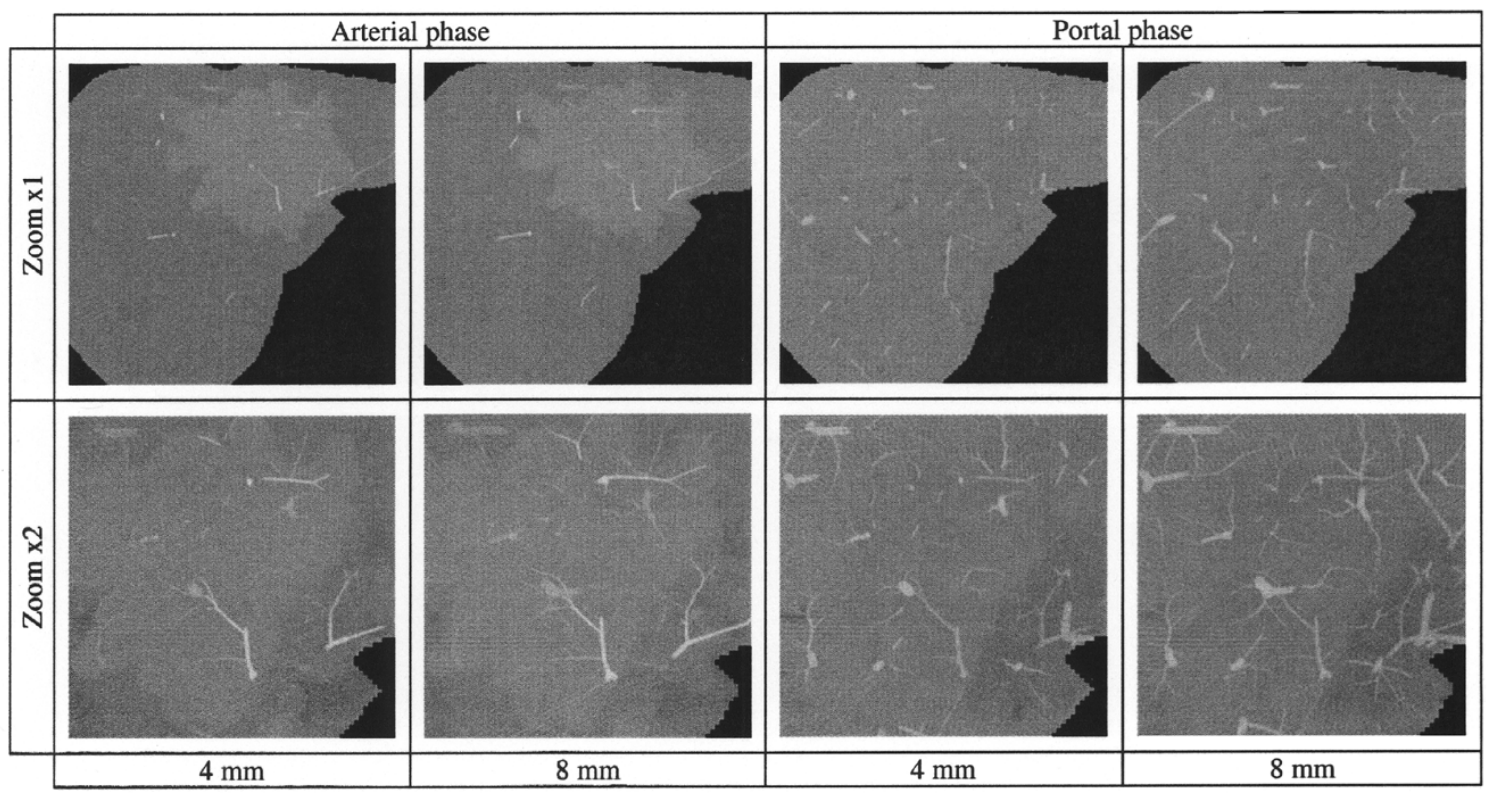

Fig. 5. Simulation of $\mathrm{CT}$ scans during the contrast medium (CM) propagation, with two spatial resolutions (zoom $\times 1, \times 2)$ and two slice thicknesses' $(4 \mathrm{~mm}, 8 \mathrm{~mm})$. The hypervascular lesion is hyperdense during the arterial phase and slightly hypodense during the portal phase.

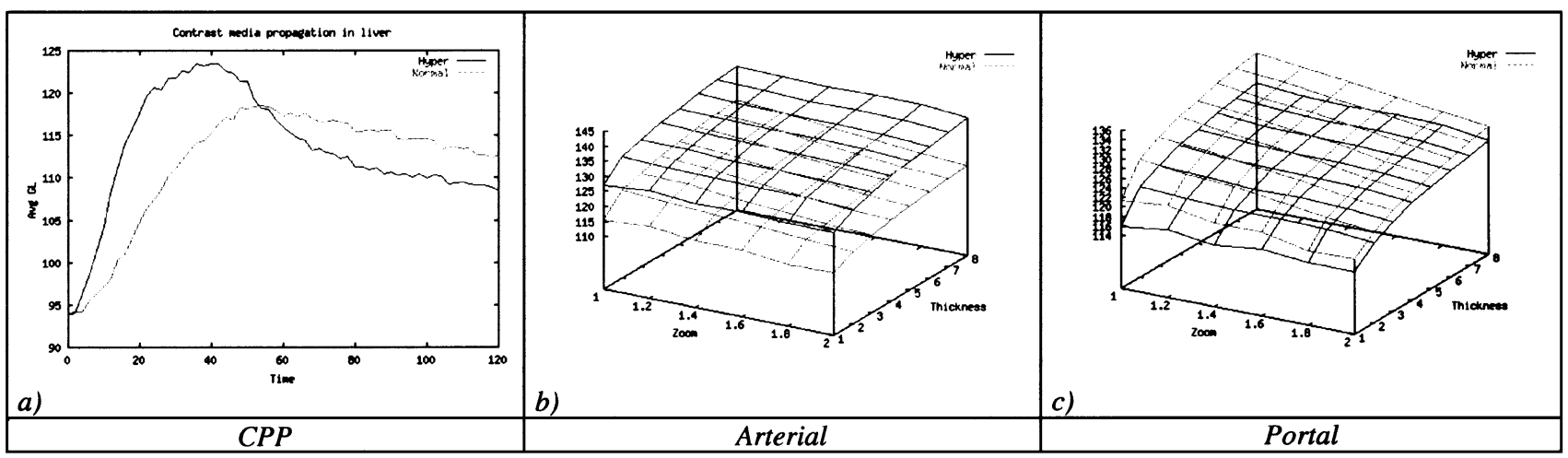

Fig. 6. Evolution of the "gray level distribution" (vertical axis) according to zoom and slice thickness and acquisition time, in normal parenchyma (dashed line) and hypervascular tumor (solid line).

the spatial resolution, the time windows and delays) on the "decision space" can be objectively anticipated. For instance, instead to make use of simple shaped phantoms with additive noise to evaluate the performances of detection, segmentation, reconstruction algorithms among others, realistic objects with varying observation conditions can lead to more robust conclusions. Appropriate protocols can then be derived in a predictive way.

To summarize, model-driven acquisition, analysis and interpretation are offered by such a generic frame. Of course, its relevance goes well beyond the imaging field here addressed. The same views can be applied to model-based signal processing [17].

\section{Analysis of Dynamic Sub Cellular Processes in IMAGE SEQUENCES}

At a lower scale, fluorescent confocal microscopy permits to observe marked subcellular structures over time, such as the chromatin or the nuclear envelope. A general objective of the researchers in different disciplines concerned with such dynamic processes is to better understand the subcellular mechanisms involved in order to arrive to a better comprehension of the cell. The knowledge about the dynamics in biological systems provides also highlights about their biophysical or biochemical condition (i.e a lack of some components in cell membranes changes the way they move). Therefore, we are looking for the extraction of meaningful quantitative parameters from image sequences and for a concise description of complex movements. A related challenge is the conception of an appropriate dynamical model for certain biological processes.

In this section, we consider an example from cell biology related to the dynamics during cell division (the breakdown of the nuclear envelope).

\section{A. Nuclear Envelope Breakdown (NEBD)}

We will summarize shortly the biological aspects related to the NEBD and its position and role in the cell division cycle. 


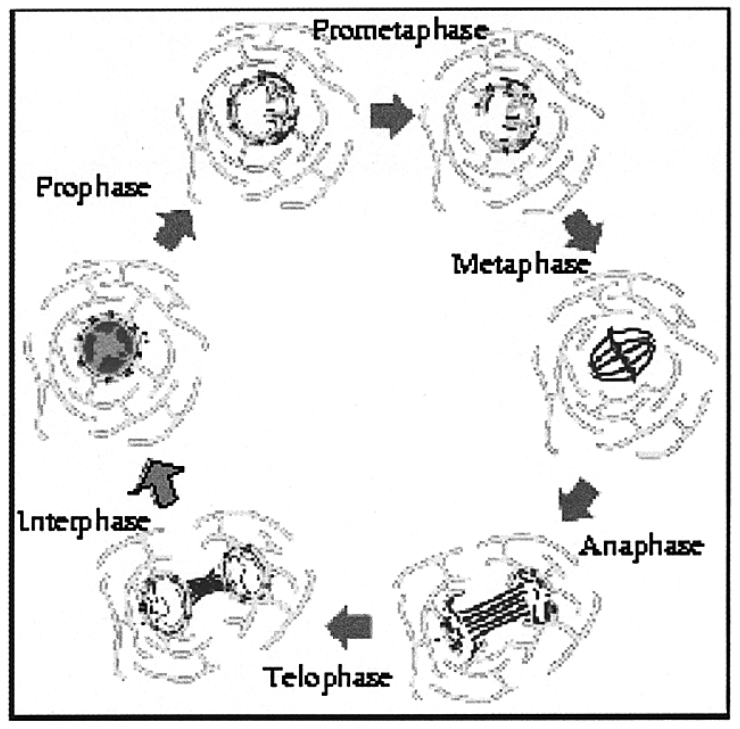

Fig. 7. Different phases in the cell division cycle related to the division of the cell nucleus (reproduced from http://www.embl-heidelberg.de/ExternalInfo/ellenberg/).

1) Mitosis: The cell cycle-from the formation of a cell (after the division of its mother cell) until its division in two daughter cells-consists of several phases, where the phases related to the division of the cell nucleus (except the replication of the chromatin) form the mitosis [1]. These temporally consecutive phases are (in their temporal order) the prophase, prometaphase, metaphase, anaphase and the telophase (Fig. 7). The breakdown of the nuclear envelope (NE) is prepared in prophase. The formation of the first hole in the envelope (constituted by an inner and outer membrane) introduces prometaphase during which the whole envelope breaks down into small fragments. Therefore, we consider in detail only these two phases of mitosis. In prophase the chromosomes condense slowly and their characteristic "X-form" begins to appear in which two sister chromatids can be distinguished. At the same time, the mitotic spindle begins to form. It is an apparatus of a number of tube-like flexible structures-called microtubules-centered in the spindle poles and able to move and to exert forces. When the first hole in the NE appears, the condensation rate of chromatin increases dramatically. Also, some of the microtubules can then enter into the nuclear region. They attach on each sister chromatid and move the chromosome to the metaphase plate.

2) A Model for the Nuclear Envelope Breakdown: A model of the NEBD has been proposed by Beaudouin et al. [7]. It is of particular interest for us as it emphasizes the part played by the biomechanical forces tearing the envelope which can be investigated by analyzing the movements of the envelope (Fig. 8). NEBD is prepared in prophase. The earliest morphological changes of the cell nucleus accompanying the entry into mitosis are invaginations in the NE at the site of the centrosomes. At the same time, it can be observed that the motor protein dynein attaches to the NE. However, a stretching and, finally, the tearing of the lamina is observed at a position distal from the centrosomes at the upper surface of the envelope (whereas

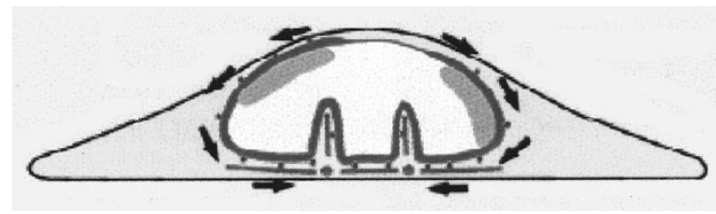

Fig. 8. Microtubule dependent forces acting on the nuclear envelope (reproduced from [2]).

the centrosomes are at its lower surface). Based on these observations, Beaudouin et al. [7] proposed a model of the nuclear envelope breakdown with microtubule dependent forces as the fundamental phenomena relating the observations. The assumption of the authors is that the forces are produced by dynein moving on the microtubules toward the microtubules' minus end situated at the centrosomes and that these forces are mediated over the whole envelope (or lamina) surface creating a tension on its upper side (Fig. 8). This tension is supposed to lead to the tearing of lamina and envelope. Another observation is that the site of tearing was always detected in an area not in contact with chromosomes. Independently, it was noticed [65] that in the areas of chromosome binding the concentration of lamin proteins is higher. After the tearing of the lamina, the gaps in the envelope expand, the lamina depolimerizes and NE fragments move toward the centrosomes, possibly in a dynein-mediated way.

\section{B. Imaging the NEBD and Motion Analysis}

A quantitative approach based on image motion analysis is required to validate and refine the above assumptions and knowledge. It can be performed with a confocal laser scanning microscope, the nuclear lamina being fluorescently labeled. A grid can then be photobleached on the surface of the lamina [49] which moves with the surface and on which the movement analysis is based. Another possibility to track the movements on the envelope surface is to image the nuclear pore complexes which permits a higher temporal resolution as no photobleaching is necessary [19]. The images are taken at different time points around the formation of the first hole.

Using the example of the nuclear envelope breakdown, some of the issues of a computer based motion analysis in image sequences can be illustrated. As we are interested not only on a special sequence of the NEBD of a single cell but on the common observations during the breakdown for different cells, a first issue is "how to compare complex movements in different sequences." This implies to find common characteristics or a common structure of the movement in different sequences and to the capability to discriminate different types of movement (i.e., normal cells or tumor cells, for instance). Further issues, directly of concern for the above model of the NEBD, must be addressed to specify if the forces are mediated globally or not, and if we can observe a continuous increase of the tension (or decrease after the formation of the first hole). Which conclusions can be drawn about the sources of the forces and about the physical properties of the lamina ? How are the forces mediated by the lamina; can a relationship be established-in a mathematically controllable 

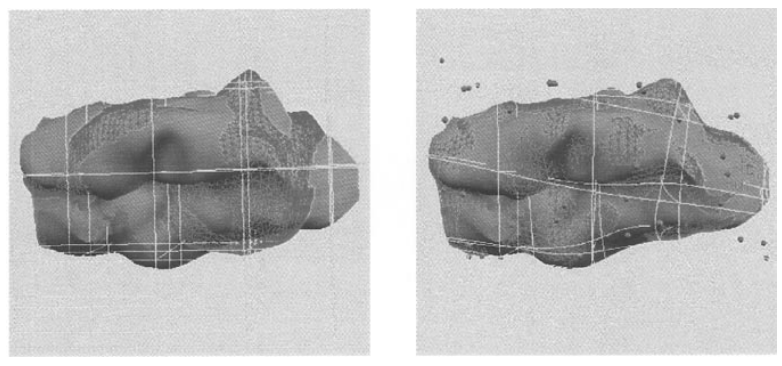

Fig. 9. Visualization of the extracted motion by using deformation grids. Reproduced from [27] with the permission of Springer-Verlag, New York.

way-between the lamin concentration and the way how the lamina moves? In order to investigate these aspects, a powerful tool would be a biomechanical model of the NEBD integrating the information extracted by image processing.

From the image processing side, two of the required basic steps are: i) to extract accurate parameters (global parameters as well as local ones) which are related to the movement; and ii) to propose a concise description of complex movement facilitating its interpretation.

Different approaches of motion estimation have been applied to the analysis of dynamic processes in biomedicine among which: i) the local determination of the optical flow based on the motion constraint equation [18], [35], [53]; ii) the tracking of segmented small particles moving individually [32], [73]; and iii) the use of parametric nonrigid registration which determines the parameters of a predefined transformation [29], [49]. All these techniques are extensively studied in the literature (reviews can be found in [11] and [53]).

Complex movements are however difficult to describe and interpret. Germain et al. [29] proposed a continuous description with the eigenvalues of the affine motion matrix extracted from two consecutive images and tried to characterize in that way differences in the movement of cells involved in the wound healing process. The motion model presented in [Fieres, 00] is aimed at associating the observed deformations and the forces causing them and their location. Another possibility is the representation of the motion in a feature space, for instance by a velocity histogram [75] or a velocity intensity image [49], where each pixel encodes the absolute value of velocity in it.

The example depicted Fig. 9 has been obtained through: i) the calculation of the registering transformation for each time step to a fixed time step of the sequence; and ii) the application of this transformation to the raw images and the writing out the transformed images. This allows to better separate global effects from more local ones and eventually to detect local motors of motion.

Most of these techniques yield a description allowing the comparison, characterization, classification of movements and therewith also an interpretation of the occurring motion. For a better understanding of the mechanisms behind the movements and for the determination of the functional relationships-leading to biophysical laws-between forces, the physical constitution of the object under consideration, and

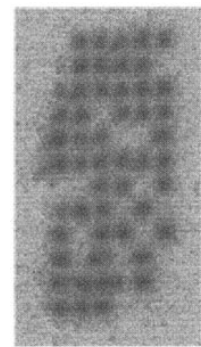

a)

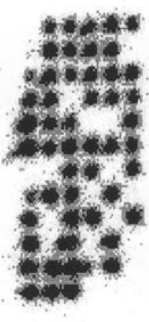

b)

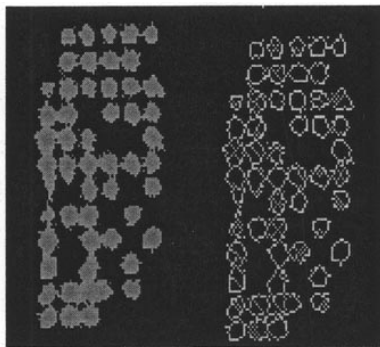

c)

d)
Fig. 10. Image coming from a Fuji bio-array device based on the $P^{33}$ isotopic activity of hybridized c-DNA's acquired by a phospho-imager ; we can see a) the raw data, each peak corresponding to a gene, b) a low-pass filtering, eliminating the high spatial frequencies, c) a watershed processing showing an over-segmentation in the water basins, and d) the contouring of the limits between water basins [49].

the observed movements, an investigation going beyond the mentioned interpretation is necessary. For instance, in the case of the lamina movements described in Section IV-A, a biophysical surface model could help to determine which forces (and their locations) are involved in an observed motion and lamin concentration. Here, the lamin protein meshwork can be cloned simulated by a model meshwork integrating finite element methods.

\section{Bio-ARray IMAging AND Genetic REgulation NETWORK MODELING}

During recent years the rapid development of bio-arrays techniques based on isotopic or fluorescent activity of hybridized DNA chips (Fig. 10) allowed the biologist to give to a gray level peak the signification of an expression rate for the genes studied in the bio-array [22]. If we repeat the bio-array acquisition at different times of the cell cycle for different cells of the same tissue, we can calculate correlations between genes expression rates and hence we are able to make explicit a matrix $W$ called the intergenic interaction matrix representing the repression or induction influences a gene can exert on other genes.

The interaction matrix $W$ is similar to the synaptic weight matrix, which rules the relationships between neurons in a neural network. An interaction between the gene $G_{j}$ and the gene $G_{i}$ is identified through the coefficient $w_{i j}$ of $W$, equal to +1 (respectively, $-1,0$ ) if the gene $G_{j}$ activates (respectively, inhibits, does not influence) the gene $G_{i}$, the state $x_{i}$ of the gene $G_{i}$ being equal to +1 (respectively, -1 ), if it is (respectively, is not) expressed. The value of $x_{i}$ is fixed by the activity level detected in the bio-array image by segmenting their peaks (Fig. 11) and integrating the gray level inside these peaks: if the integrated activity of the $i$ th peak is sufficiently high, then $x_{i}=1$; if not, then $x_{i}=0$. In the case of small genetic regulatory networks (the smallest ones are called operons and the first historically studied is the lactose operon), the knowledge of such a matrix $\mathrm{W}$ permits to explicit all possible stationary behaviors of the organizms having the corresponding genome. The change of 


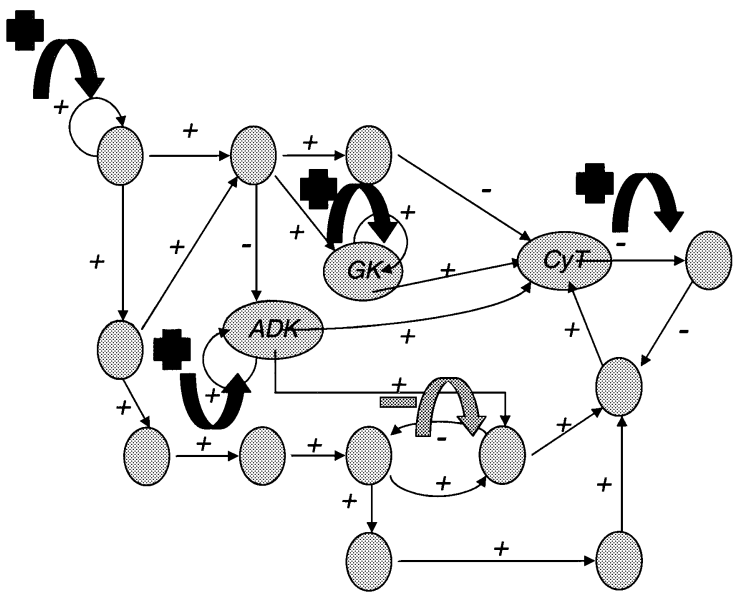

Fig. 11. Gastrulation operon graph showing the positive and negative interactions between the genes involved at this step of the embryogenesis; the loops (four positive and one negative) are indicated by thick arrows (from [2] and [44]).

state of gene $G_{i}$ between times $t$ and $t+1$ obeys a threshold rule: $x_{i}(t+1)=H\left(\sum_{j=1, n} w_{i j} x_{j}(t)-b_{i}\right)$ or in vectorial form: $\mathbf{x}(t+1)=H(W \mathbf{x}(t)-\mathbf{b})$, where $H$ is the Heaviside step function between -1 and $+1(H(y)=1$, if $H(y)=-1$ if $y<0$ ), and the $b_{i}$ 's are threshold values. The values of the parameters $w_{i j}$ 's and $b_{i}$ 's are calculated from the bio-array image. For calculating the $w_{i j}$ 's, we can determine the s-directional correlation $\rho_{i j}(s)$ between the state vector $\left\{x_{j}(t-s)\right\}_{t_{\in} C}$ of gene $G_{j}$ at time t-s and the state vector $\left\{x_{i}(t)\right\}_{t_{\in} C}$ of gene $G_{i}$ at time $t, t$ varying during the cell cycle $C$ supposed of length $M$ and corresponding to observation times of the bio-array images

$$
\rho_{i j}(s)=\frac{\left(\frac{\sum t_{\in} c x_{j}(t-s) X_{i}(t)-\sum t \in c x_{i}(t-s) \sum t \in c x_{i}(t)}{M}\right)}{\sigma_{i}(s) \sigma_{i}(O)}
$$

where

$\sigma_{j}(s)=\sum t \in C X_{j}(t-s)^{2}\left(\sum t \in C x_{j}(t-s)\right)^{2} / M^{1 / 2}$

and then take

$$
w_{i j}=\operatorname{sign}\left(\sum s=1, \ldots k \rho_{i j}(s) / k\right)
$$

if $\left|w_{i j}\right| \geq \eta ; w_{i j}=0$, if $\left|w_{i j}\right|<\eta$, $\eta$ being a decorrelation threshold.

When $t$ is increasing, the genes states reach a stable set of configurations (fixed configuration or cycle of configurations) called attractors of the genetic network dynamics. For example, in the regulatory network ruling Arabidopsis thaliana flower morphogenesis, the interaction matrix $\mathrm{W}$ is a $(11,11)$-matrix with only 22 nonzero coefficients [52]. This matrix presents $P(W)=4$ positive loops (i.e., its incidence graph $G(W)$ has four closed paths starting with a gene and coming back to it after an even number of inhibitions) and $A(W)=4$ attractors. It is in general of great biological interest and relevance to determine matrices having characteristic properties like: 1) a minimal number of non zero coefficients for a given set of attractors (fixed points or cycles); or 2) a minimal number $P(W)$ of positive loops, controlling the number $A(W)$ of attractors and their stability (cf. [23], [31], [58], and [64] for results in the continuous case, and [15] for the discrete one).

When it is impossible to obtain all coefficients of $W$ (neither from literature nor from bio-array calculations), we can complete $W$. We choose randomly the missing coefficients by respecting the connectivity coefficient $K(W)=I / N$, ratio between the number $I$ of interactions and the number $N$ of genes, and the mean inhibition weight $I(W)=R / I$, ratio between the number of inhibitions (or repressions) $R$ and $I . K(W)$ is in general between 1.5 and 3 and $I(W)$ between $1 / 3-2 / 3$, for many known regulatory networks (lactose or phage $\lambda$ lytic operons, gastrulation, i.e., digestive tube embryogenesis, or Arabidopsis thaliana flowering networks, etc.).

If $G(W)$ is the incidence graph associated to $W$, then we call connected component of $G(W)$ each set of genes such that there is a path between every pair of genes along a sequence of arcs in $G(W)$. A garden of Eden is a gene receiving no arc, but influencing at least one other gene. A regulon is a connected component of $G(W)$ having exactly one positive autocatalysis (i.e., a loop from a gene to itself) and one negative loop, these loops sharing the autocatalyzed gene. Such a regulon is present for example in the lactose operon.

In 1948, Delbrück [20] conjectured that positive loops in the interaction graph of a regulatory network was a necessary condition for the cell differentiation, i.e., for the existence of multiple attractors of the genes expression; this conjecture has been written in a good mathematical context by Thomas in 1981 [71]. The presence of positive loops is related to the observation of multiple attractors, which definitively gives to the positive loop another signification that to the negative loop, more related to the stability of the system (like in the classical Watt regulator, well known in cybernetics). In 1992, Kauffman [41] conjectured that the mean number of attractors for a Boolean genetic network with $\mathrm{n}$ genes and connectivity $K(W)=2$, was of order of $\sqrt{ } n$ [21]. This conjecture is now supported by real observations: we have about 30000 genes in the human genome and about 200 different tissues, which can be considered as different attractors of the same dynamics. For Arabidopsis thaliana, there is $A(W)=4 \approx \sqrt{ } 11$ different tissues (sepals, petals, stamens and carpels) [21] and for the lytic operon of the phage $\lambda, K(W)=14 / 5=2.8, I(W)=9 / 14$ and there is $A(W)=2 \approx \sqrt{ } 5$ (lytic and lysogenic) attractors [70].

If we consider the network ruling the gastrulation in Drosophila (cf. Fig. 11 and [2], [44]), it is easy to check that $K(W)=25 / 15, I(W)=5 / 15, P(W)=4$ and $A(W)=2$ (the corresponding cells being the ordinary ectoderm cells, for the skin, and the trapezoidal cells, for the intestine). The regulation graph contains five connected components (whose three are singletons). In this case, the classical Kolmogorov-Rashevski-Turing models of reaction-diffusion [42], [60], [72] are well explaining the epigenetic part of the start of the gastrulation, but only after the apparition of a new trapezoidal cell presenting an apical constriction due to the change of intracellular energetic balance due to the expression of the genes A [adenylate kinase (ADK)] and $\mathrm{N}$ [nucleoside diphosphate kinase (NDK)], and to their 
action on the cytoskeleton genes $\mathrm{C}$. This is explained by a change of attractor basin by the trapezoidal cell starting the gastrulation process due to the genetic regulation pathway of the Fig. 11. This regulatory network is now confirmed by the genes expression calculated from bio-array imaging as explained above.

From a general standpoint, simulations of the model above allow the biologist to pay attention for the crucial parameters to acquire in bio-array imaging. The notion of crucial parameters comes from the classical proposal by Popper [59] for a general modeling approach.

1) Propound empirically testable model $M$ with measurable variables and parameters

2) Aim to refute them by comparing parameters critical values calculated in the model $M$ (e.g., causing a qualitative change in the dynamics, as the passage from a stationary state to an oscillatory one) and measured in the experiment

3) If $M$ is refuted, aim to replace it by another model $M^{\prime}$ more general and precise (i.e., with higher information content), explaining the refuting evidence of $M$ and moreover independently testable.

The few examples reported in this paper are far from describing the wide spectrum of applications and complex mixed problems to be addressed for image processing and shape modeling in morphogenesis. It is likely that many attempts will be devoted for instance to evaluate tumor growth duration and initial starting localization (with inverse dynamics studies based on microscopic and NMR imaging), to track cells in wound healing process and modeling cells migration, or to explain mitochondrial inner membrane building in the adaptation to effort and aging.

\section{CONCLUSION AND Future ChallengeS}

The combined modeling, acquisition and processing approaches advocated for several future demands: increasing the interest for physiological modeling as such and its coupling with image analysis methods. The complexity of the living systems (which can be represented by the number of differential equations, the number of parameters or unmeasured variables, etc.), the theoretical limits to deal with can not prevent this effort. Any spatio-temporal scale of observation and modeling of nonlinear, deterministic or stochastic, multivariate systems will provide significant clues on the underlying interactions and behaviors and sketch new features of the biological picture. It is our belief that the wide range of model-driven applications (from experiment guiding, data acquisition setting, problem solving to process understanding, etc.), already visible in most areas, is an enough strong motivation for engineering researchers. Pluridisciplinarity is going more and more critical to balance the trend toward highly specialized fields and to make the best benefits of their impressive advances. It is clearly a challenge because mathematics, physics, physiology, biology, and others are concerned. However, image processing, with its capability to efficiently extract information from data, can not get away from this challenge. Data and model fusion is a natural frame in which integrative physiology can be soundly stated if a real implication into physiological mechanisms is achieved. This joint effort must be associated to new initiatives able to answer to the clinical requirements (patient-based fitting, international data sets, large scale evaluation) and closely linked with the breakthroughs emerging from micro-arrays, integrated sensing-actuating devices, etc., which will determine the nature, scale, and quality of observations, all elements critical for any improvements in information processing.

\section{REFERENCES}

[1] B. Alberts, D. Bray, A. Johnson, J. Lewis, M. Raff, K. Roberts, and P. Walter, Essential Cell Biology. An Introduction to the Molecular Biology of the Cell. New York: Garland, 1998.

[2] J. Aracena, "Modèles mathématiques discrets associés à des systèmes biologiques," Ph.D. dissertation, Univ. Chile, Santiago, Chile, 2001.

[3] S. Audoly, G. Bellu, L. D'Angio, M. P. Saccomani, and C. Cobelli, "Global identifiability of nonlinear models of biological systems," IEEE Trans. Biomed. Eng, vol. 48, pp. 55-65, Jan. 2001.

[4] R. L. Baron, "Understanding and optimizing use of contrast material for CT of the liver," AJR, vol. 163, pp. 323-331, 1994.

[5] J. B. Bassingthwhaite, H. Qian, and Z. Li, "The cardiome project: An integrated view of cardiac metabolism and regional mechanical function," Adv. Experiment. Med. Biol., vol. 471, pp. 541-553, 1999.

[6] W. Baumeister, R. Grimm, and J. Walz, "Electron tomography of molecules and cells," Trends Cell Biol., vol. 9, pp. 81-85, 1999.

[7] J. Beaudouin, D. Gerlich, N. Daigle, R. Eils, and J. Ellenberg, "Nuclear envelope breakdown proceeds by microtubule-induced tearing of the lamina," Cell, vol. 108, pp. 83-96, 2002.

[8] J. Bézy-Wendling and M. Kretowski, "Modeling hepatic enhancement in computer tomography for texture interpretation," presented at the IEEE Int. Symp. Biomedical Imaging, Washington, DC, July 7-10, 2002.

[9] J. Bézy-Wendling, M. Kretowski, and Y. Rolland, "Hepatic tumor enhancement in computer tomography: Combined models of liver perfusion and dynamic imaging," Comput. Biol. Med., vol. 33, pp. 77-89, 2003, to be published.

[10] J. Bézy-Wendling, M. Kretowski, Y. Rolland, and W. Le Bidon, "Toward a better understanding of texture in vascular CT scan simulated image," IEEE Trans. Biomed. Eng., vol. 48, pp. 120-124, Jan. 2001.

[11] L. G. Brown, A Survey of Image Registration Techniques. Washington, DC: ACM Computing Surveys, 1992, vol. 24, pp. 325-376.

[12] A. Bruno, R. Collorec, J. Bézy-Wendling, P. Reuzé, and Y. Rolland, "Texture analysis in medical imaging," in Contemporary Perspectives in Three-Dimensional Biomedical Imaging, C. Roux and J. L. Coatrieux, Eds. Amsterdam, The Netherlands: IOS Press, 1997, pp. 133-164.

[13] R. E. Carson and C. Cobelli, Modeling, Methodology for Physiology and Medicine. New York: Academic, 2000.

[14] A. Chu, C. M. Sehgel, and J. F. Greenleaf, "Use of gray value distribution of run lengths for texture analysis," Pattern Recogn. Lett., vol. 11, pp. 415-420, 1990.

[15] O. Cinquin and J. Demongeot, "Positive and negative feedback: Mending the ways of sloppy systems," C. R. Acad. Sci. Biol., vol. 325, pp. 1085-1095, 2002.

[16] - "Positive and negative feedback: Striking a balance between necessary antagonists," J. Theor. Biol., vol. 216, pp. 229-241, 2002.

[17] J. L. Coatrieux, "Issues on signal processing and physiological modeling," Crit. Rev. Biomed. Eng., vol. 30, pp. 9-56, 2003, to be published.

[18] J. L. Coatrieux, C. Toumoulin, and R. Collorec, "3D cardiac modeling: A computer approach in spatio-temporal image sequences," J.Biol. Syst., vol. 4, no. 2, pp. 181-197, 1996.

[19] N. Daigle, J. Beaudouin, L. Hartnell, G. Imreh, E. Hallberg, J. Lippincott-Schwartz, and J. Ellenberg, "Nuclear pore complexes form immobile networks and have a very low turnover in live mammalian cells," J. Cell Biol., vol. 154, pp. 71-84, 2001. 
[20] M. Delbrück, "Discussion, in: Unités biologiques douées de continuité génétique," in Colloques Int. CNRS, vol. 8, 1949, pp. 33-35.

[21] J. Demongeot, J. P. Françoise, M. Richard, F. Senegas, and T. P. Baum, "A differential geometry approach for biomedical image processing," C. R. Acad. Sci. Biol., vol. 325, pp. 367-374, 2002.

[22] J. Demongeot, "Modeling the genetic expression: Positive interaction loops and genetic regulatory systems," in Proc. Mathématiques et Biologie, Société Mathématique de France, Paris, France, 2002, pp. 67-94.

[23] , "Multi-stationarity and cell differentiation," J. Biol. Syst., vol. 6, pp. 1-2, 1998

[24] G. D. Dodd and R. Baron, "Investigation of contrast enhancement in CT of the liver: The need for improved methods," AJR, vol. 160, pp. 643-646, 1993.

[25] N. Emptage, "Fluorescent imaging in living systems," Current Opinions Pharmacol., vol. 1, pp. 521-525, 2001.

[26] J. Fessler, "Statistical methods for image reconstruction, biomedical imaging V," presented at the 5th IEEE EMBS Int. Summer School Biomedical Imaging, 2002.

[27] J. Fieres, J. Mattes, and R. Eils, "A point set registration algorithm using a motion model based on thin-plate splines and point clustering," in Pattern Recognition 2001, B. Radig and S. Florczyk, Eds. Munich, Germany: Springer-Verlag, 2001, vol. 2191, Lecture Notes in Computer Science (LNCS), pp. 76-83.

[28] M. G. Frederick, B. L. McElaney, A. Singer, K. S. Park, E. K. Paulson, S. G. McGee, and R. C. Nelson, "Timing of parenchymal enhancement on dual-phase dynamic helical CT of the liver: How long does the hepatic arterial phase predominates?," AJR, vol. 166, pp. 1305-1310, 1996.

[29] F. Germain, A. Doisy, X. Ronot, and P. Tracqui, "Characterization of cell deformation and migration using a parametric estimation of image motion," IEEE Trans. Biomed. Eng., vol. 46, pp. 584-600, May 1999.

[30] B. Gilbert, G. Margaritondo, D. Mercanti, T. Casalbore, and G. De Stasio, "Synchrotron spectromicroscopy in medicine and biology," J. Alloys Compounds, vol. 328, pp. 8-13, 2001.

[31] J. L. Gouzé, "Positive and negative circuits in dynamical systems," J. Biol. Syst., vol. 6, pp. 11-16, 1998.

[32] I Grant and Editor, Eds., Selected Papers on Particle Image Velocimetry. Bellingham, WA: SPIE Press MS99, 1994.

[33] A. Hengerer and T. Mertelmeier, "Molecular biology for medical imaging," Electromedica, vol. 69, no. 1, pp. 2-7, 2001.

[34] G. T. Herman, Image Reconstruction From Projections. New York: Academic, 1980

[35] B. Horn and B. G. Shunck, "Determining optical flow," Art. Intell., vol. 17, pp. 185-203, 1981.

[36] L Ibanez, E. Hamitouch, M Boniou, and C Roux, "Morphogenesisbased deformable models. Application to 3D image segmentation and analysis," in Proc. MICCAI 2001, vol. LNCS 2208, W. Niessen and M. Viergever, Eds., 2001, pp. 1369-1370.

[37] R. E. Jacobs and S. R. Cherry, "Complementary emerging techniques: High resolution PET and MRI," Current Opinions Neurobiol., vol. 11, pp. 621-619, 2001.

[38] J. Jacquez, Compartmental Analysis in Biology and Medicine. Ann Arbor, MI: BioMedware, 1996.

[39] Z. Kam, E. Zamir, and B. Geiger, "Probing molecular processes in live cells by quantitative multidimensional microscopy," Trends Cell Biol., vol. 11, pp. 329-334, 2001.

[40] R. Karch, F. Neumann, M. Neumann, and W. Schreiner, "A three-dimensional model for arterial tree representation, generated by constrained constructive optimization," Comput. Biol. Med., vol. 29, pp. 19-38, 1999.

[41] S. Kauffman, The Origins of Order. Oxford, U.K.: Oxford Univ. Press, 1993.

[42] A. N. Kolmogorov, I. Petrowski, and N. Piscounov, "Etude de l'équation de la diffusion avec croissance de la quantité de matière et son application à un problème biologique," Moscow Univ. Bull. Math., vol. 1, pp. 1-25, 1937.

[43] M Kretowski, Y. Rolland, J. Bézy-Wendling, and J. L. Coatrieux, "Fast algorithm for 3-D vascular tree modeling," Comput. Meth. Progr. Biomed., vol. 70, pp. 129-136, 2003, to be published.

[44] M. Leptin, "Gastrulation in drosophila: The logic and the cellular mechanisms," EMBO J., vol. 18, pp. 3187-3192, 1999.

[45] Z.-P. Liang and P. C. Lauterbur, Principles of Magnetic Resonance Imaging. Piscataway, NJ: IEEE Press, 1999.
[46] L. Ljung and S. T. Glad, "On global identifiability for arbitrary model parametrizations," Automatica, vol. 30, no. 2, pp. 265-276, 1994.

[47] A. Y. Louie, M. M. Huber, E. T. Ahrens, U. Rothbacher, R. E. Jacobs, S. E. Fraser, and T. J. Meade, "In-vivo visualization of gene expression using magnetic resonance imaging," Nature Biotechnol., vol. 18, pp. 321-325, 2000.

[48] R. Materne, B. E. Van Beers, A. M. Smith, I. Leconte, J. Jamart, J. P. Dehoux, A. Keyeux, and Y. Hormans, "Non-invasive quantification of liver perfusion with dynamic computed tomography and a dual input one-compartmental model," Clinical Sci. '99, pp. 517-525, 2000

[49] J. Mattes, J. Fieres, J. Beaudouin, D Gerlich, J. Ellenberg, and R. Eils, "New tools for visualization and quantification in dynamic processes: Application to the nuclear envelope dynamics during mitosis," in Proc. MICCAI 2001, vol. LNCS 2208, 2001, pp. $1323-1325$.

[50] J. Mattes and J. Demongeot, "Efficient algorithms to implement the confinement tree," in Proc. DGCI 2000, vol. LNCS 1953, G. Borgefors, I. Nyström, and G. Sanniti di Baja, Eds., 2000, pp. 392-405.

[51] J. Mattes, M. Richard, and J. Demongeot, Tree Representation for Image Matching and Object Recognition, ser. Lecture Notes in Computer Science. New York: Springer-Verlag, 1999, vol. 1568, pp. 298-309.

[52] L. Mendoza and E. R. Alvarez-Buylla, "Dynamics of genetic regulatory network for A. Thaliana flower morphogenesis," J. Theoret. Biol., vol. 193, pp. 307-319, 1998.

[53] A. Mitiche and P. Bouthemy, "Computation and analysis of image motion: A synopsis of current problems and methods," Int. J. Comput. Vision, vol. 19, pp. 29-55, 1996.

[54] J. D. Murray, Mathematical Biology. New York: Springer-Verlag, 1993.

[55] V Ntziachristos, J. Ripoll, and R. Weissleder, "Would near-infrared fluorescence signals propagate through large human organs for clinical studies?," Opt. Lett., vol. 27, pp. 333-335, 2002.

[56] M. Phelps, A. Chatziioannou, S. Cherry, and S. Gambhir, "Molecular imaging of biological processes from micro PET in mice to PET in patients," presented at the IEEE Int. Symp. Biomedical Imaging, Washington, DC, July 2002.

[57] M. E. Phelps, "PET: The merging of biology and imaging into molecular imaging," J. Nucl. Med., vol. 41, no. 4, pp. 661-681, 2000.

[58] E. Plahte, T. Mestl, and S. W. Omholt, "Feedback loops, stability and multi-stationarity in dynamical systems," J. Biol. Syst., vol. 3, pp. 409-414, 1995.

[59] K. R. Popper, The Logic of Scientific Discovery. London, U.K.: Hutchinson, 1959.

[60] N. Rashevsky, Mathematical Biophysics. Cambridge, U.K.: Cambridge Univ. Press, 1948.

[61] C Roux and J. L. Coatrieux, Contemporary Perspectives in ThreeDimensional Biomedical Imaging. Amsterdam, The Netherlands: IOS Press, 1997.

[62] J. A. Sethian, "Level set methods and fast marching methodsevolving interfaces in computational geometry, fluid mechanics," in Computer Vision and Materials Science. Cambridge, U.K.: Cambridge Univ. Press, 1999.

[63] G. Slodzian, M. Chaintreau, R. Dennebouy, and A. Rousse, "Precise in situ measurements of isotopic abundances with pluse counting of sputtered ions," Eur. Ohys. J., vol. 14, pp. 199-231, 2001.

[64] E. H. Snoussi, "Necessary condition for multi-stationarity and stable periodicity," J. Biol. Syst., vol. 6, pp. 3-10, 1998.

[65] N. Stuurman, S. Heins, and U. Aebi, "Nuclear lamins: Their structure, assembly, and interactions," J. Struct. Biol., vol. 122, pp. 42-66, 1998.

[66] J. Tabony, N. Glade, C. Papaseit, and J. Demongeot, "The effect of gravity on microtubule self-organization," J. Phys. IV, vol. 11, pp. 239-246, 2001.

[67] R. Taylor, S. Lavallée, G. Burdea, and R. Mosges, Computer-Integrated Surgery: Technology and Clinical Applications. Cambridge, MA: MIT Press, 1996.

[68] D Terzopoulos, "Deformable and functional models: Theory and prospects, in biomedical imaging IV," presented at the 4th IEEE EMBS Summer School Biomedical Imaging, C. Roux and J. L. Coatrieux, Eds., 2000.

[69] D. Terzopoulos, A. Witkin, and M. Kass, "Constraints on deformable models: Recovering 3D shape and nonrigid motion," Art. Intell., vol. 36, no. 1, pp. 91-123, 1988. 
[70] D. Thieffry, M. Colet, and R. Thomas, "Formalization of regulatory networks," Math. Model. Sci. Comput., vol. 2, pp. 144-151, 1993.

[71] On the Relation Between the Logical Structure of Systems and Their Ability to Generate Multiple Steady States or Sustained Oscillations, ser. Springer Series in Synergetics, vol. 9, pp. 1-23, 1981.

[72] A. Turing, "The mathematical basis of morphogenesis," Phil. Trans. Roy. Soc., vol. B237, pp. 37-47, 1952.

[73] W. Tvaruskó, M. Bentele, T. Misteli, C. Kaether, D. L. Spector, H. H. Gerdes, and R. Eils, "Time-resolved analysis and visualization of dynamic processes in living cells," PNAS, vol. 96, pp. 7950-7955, 1999.

[74] M. Unser and Z. P. Liang, presented at the IEEE Int. Symp. Biomedical Imaging: From Macro to Nano, Washington, DC, July 2002.

[75] D. Uttenweiler, C. Veigel, R. Steubing, C. Götz, S. Mann, H. Haussecker, B. Jähne, and R. Fink, "Motion determination in actin filament fluorescence images with a spatio-temporal orientation analysis method," Biophys. J., vol. 78, pp. 2709-2715, 2000.

[76] R. Weissleder, "Scaling down imaging: Molecular mapping of cancer in mice," Nature Rev. Cancer, vol. 2, pp. 11-18, 2002.

[77] —, "In vivo imaging of tumors with protease-activated near-infrared fluorescent probes," Nature Biotechnol., vol. 17, no. 4, pp. 375-378, 1999. 